CERN-TH.7552/95

OUTP 94-38P

RAL-95-002

January 1995

\title{
An analytic solution of the BFKL equation with momentum cutoffs
}

\author{
M.F.McDermott ${ }^{1}$ \\ J.R.Forshaw ${ }^{2}$ \\ and \\ G.G.Ross ${ }^{1}$ \\ 1 Department of Theoretical Physics, University of Oxford, \\ 1-4 Keble Road, Oxford, OX1 3NP, United Kingdom. \\ 2 Rutherford Appleton Laboratory, \\ Chilton, Didcot, Oxon, OX11 0QX, United Kingdom.
}

\begin{abstract}
We outline a general method for obtaining the solution to the $(t=0)$ BFKL equation in the presence of transverse momentum cutoffs. A lower cutoff allows one to avoid integration over nonperturbative momenta and an upper one is needed from energy-momentum conservation. Our method allows for the inclusion of an arbitrary number of poles in the kernel and is applicable to any input distribution. Taking Mellin transforms, we discuss the effect of introducing cutoffs by considering the singularity structure in the transform space. We present an improved calculation of the small- $x$ slope of the gluon density.
\end{abstract}




\section{Introduction}

Recent results from the HERA electron-proton collider on the small- $x$ rise of the nucleon structure function $F_{2}\left(x, Q^{2}\right)$ [1], 2] have generated considerable theoretical interest in small- $x$ physics. Theoretical opinion on the cause of this increase is somewhat divided [3, 4, 5]. One explanation is that HERA has become sensitive to a new regime of perturbative QCD, i.e. the 'small- $x$ limit' defined by

$$
\begin{gathered}
\ln \left(\frac{1}{x}\right) \gg 1 \\
\alpha_{s} \ln \left(\frac{1}{x}\right)
\end{gathered}
$$

Generally, $x \sim Q^{2} / s$ where $Q^{2} \gg \Lambda_{Q C D}^{2}$ is some hard scale and $s$ is the process centre-of-mass energy. In the case of deep inelastic scattering, $x$ is the Bjorken- $x$ and $Q^{2}$ is the photon virtuality.

Within this regime an equation has been derived, the BFKL equation [4], which sums those terms in the perturbative expansion which have equal powers of $\alpha_{s}$ and $\ln (1 / x)$. In this limit, the strong coupling is not renormalised and remains fixed. The BFKL equation (after angular averaging) can be expressed as an integral equation for the evolution of the unintegrated gluon distribution function $f\left(x, k_{\perp}^{2}\right)$, for momentum transfer squared $t=0$ it is given by

$$
\frac{\partial f\left(x, k_{\perp}^{2}\right)}{\partial \ln 1 / x}=\left(\frac{3 \alpha_{s} k_{\perp}^{2}}{\pi}\right) \int_{0}^{\infty} \frac{d k_{\perp}^{\prime 2}}{k_{\perp}^{\prime 2}}\left\{\frac{\left(f\left(x, k_{\perp}^{2}\right)-f\left(x, k_{\perp}^{2}\right)\right)}{\left|k_{\perp}^{\prime 2}-k_{\perp}^{2}\right|}+\frac{f\left(x, k_{\perp}^{2}\right)}{\sqrt{k_{\perp}^{4}+4 k_{\perp}^{\prime 4}}}\right\} .
$$

Given an input distribution, $f\left(x_{0}, k_{\perp}^{2}\right.$ ) (which we take to be independent of $x$ ), eq.(2) produces the distribution at lower $x, f\left(x, k_{\perp}^{2}\right)$.

Although the equation embodies the complete leading $\alpha_{s} \ln x$ summation it is clear to see that there are potential difficulties associated with the infra-red and ultra-violet regions of the (semi-infinite) integration over $k_{\perp}^{2 \prime}$ in eq.(2). For small values of $k_{\perp}^{2 \prime}$, we expect the corrections to the leading log resummation to be important, e.g. the introduction (by hand) of the QCD running coupling, evaluated at the scale $k_{\perp}^{2}$, produces a logarithmic infra-red divergence in eq.(2). By introducing an infra-red cutoff one can restrict the calculation to contributions 
from the region where perturbation theory should safely apply. We may also want to introduce an upper cutoff on $k_{\perp}^{2 \prime}$ for reasons of energy conservation [6, 7]. This is a necessary ingredient if one is to satisfy energy conservation but certainly not sufficient when one sums over an infinite number of gluons. We will return to a discussion of this question later.

For a discussion and a numerical investigation of the effects of introducing cutoffs on the BFKL equation see [5, 0, 8]. These papers conclude that eq.(2) predicts a contribution to the gluon distribution which rises like $x^{-K_{0}}$ up to powers of $\ln x$, (where $K_{0}=12 \alpha_{s} \ln 2 / \pi$ ), i.e. that the small $x$ slope is relatively stable to infra-red (and ultra-violet) cutoffs. However, they also conclude that the normalisation of this contribution is uncertain since it depends on the details of the treatment of the infra-red region. Consequently, an exact prediction for the contribution of the BFKL component to $F_{2}\left(x, Q^{2}\right)$ is not possible.

Here we consider whether one can provide an analytic solution to the BFKL equation with momentum cutoffs. This has an advantage over numerical solutions in that it does not require specification of the input distribution. Moreover the effects of the cutoffs will be manifest. Our work is closely related to that of Collins and Landshoff [6] but provides a different form for the solution which is more easily generalised to deal with the exact kernel of the BFKL equation. It also provides a formalism that allows us to investigate some of the implications of energy conservation not dealt with by the momentum cutoff.

In a physical gauge two types of graph lead to equal powers of $\alpha_{s}$ and $\ln (1 / x)$ : ladder graphs involving gluons with strong ordering in $x$ up the ladder (the $f\left(x, k_{\perp}^{2 \prime}\right)$ terms in eq.(2) ) and a subclass of the possible virtual corrections to these graphs which reggeize the t-channel gluons $\left(f\left(x, k_{\perp}^{2}\right)\right.$ terms). Following the work of Collins and Landshoff [6], we restrict ourselves to introducing cutoffs on the real graphs only. This makes the problem more tractable and ensures a gauge invariant result.

The equation is best solved by taking Mellin transforms with respect to $x$ and $k_{\perp}^{2}$, solving the equation in double Mellin transform space (DMT-space), then inverting the solution back to $\left(x, k_{\perp}^{2}\right)$-space. The kernel of the BFKL equation 
has poles in the left and right $\omega$-plane, $\omega$ being the transform variable conjugate to $k_{\perp}^{2}$. We present a method for deriving the DMT gluon distribution in the cases where infra-red and/or ultra-violet cutoffs are imposed upon the real terms (the $\left.f\left(x, k_{\perp}^{2 \prime}\right)\right)$ in eq.(2). Our method is valid for any input distribution and can include any number of the poles associated with the kernel. To illustrate the method we give two examples, which we compare directly with the results of ref.[6]. In the example where both infra-red and ultra-violet cutoffs are present, we do not agree with the results of ref. [6] (we differ by a relative minus sign between terms). We discuss the effects upon the small- $x$ behaviour of the gluon distribution function due to the introduction of such cutoffs.

\section{The Pole Projection Method}

Mellin transforms with respect to $x$ and $k_{\perp}^{2}$ are defined by

$$
\begin{aligned}
f\left(x, k^{2}\right) & =\int_{c-i \infty}^{c+i \infty} \frac{d \omega}{2 \pi i}\left(k^{2}\right)^{\omega+\frac{1}{2}} \tilde{f}(x, \omega) \\
\tilde{f}(x, \omega) & =\int_{0}^{\infty} d k^{2}\left(k^{2}\right)^{-\frac{3}{2}-\omega} f\left(x, k^{2}\right) \\
\tilde{f}(x, \omega) & =\int_{c-i \infty}^{c+i \infty} \frac{d N}{2 \pi i} x^{N} \mathcal{F}(N, \omega) \\
\mathcal{F}(N, \omega) & =\int_{0}^{1} d x x^{-1-N} \tilde{f}(x, \omega) .
\end{aligned}
$$

The contours in eqs.(3, 5) lie parallel to the imaginary axis and their position is chosen such that the respective inverses exist. The addition of $1 / 2$ to the power of $k^{2}$ included in eq.(3) is merely for convenience.

In DMT-space the solution to the BFKL equation without momentum cutoffs is given by a simple geometric sum:

$$
\mathcal{F}(N, \omega)=\sum_{n=0}^{\infty}\left(\frac{-K(\omega)}{N}\right)^{n} \mathcal{F}_{0}(N, \omega)=\frac{1}{1+N^{-1} K(\omega)} \mathcal{F}_{0}(N, \omega)
$$

where $K(\omega)$ are the eigenvalues of the kernel of eq.(2),

$$
K(\omega)=\frac{3 \alpha_{s}}{\pi}\left[-2 \gamma-\psi\left(\frac{1}{2}+\omega\right)-\psi\left(\frac{1}{2}-\omega\right)\right]
$$

corresponding to the eigenfunctions $\left(k_{\perp}^{2}\right)^{1 / 2+\omega}$. The quantities $\psi$ and $\gamma$ are the log derivative of the Euler gamma function and the Euler gamma constant, respectively. 
The eigenvalues given by eq.(8) have an infinite set of simple poles at $\omega=$ $\{ \pm 1 / 2, \pm 3 / 2, \cdots\}$ as a result of the poles in the $\psi$ functions. The contour in the transform definition eq.(3) is chosen to lie midway between these poles and parallel to the imaginary axis. We can expand $K(\omega)$ in terms of these poles

$$
K(\omega)=\sum_{i}^{\infty} \frac{a_{i}}{\omega-\omega_{i}}+\sum_{j}^{\infty} \frac{b_{j}}{\omega+\omega_{j}}+h(\omega)
$$

where $h(\omega)$ is an analytic function. For an analytic solution we will approximate the infinite series by including only a finite number of these poles (bearing in mind that the integral defined in eq.(3i) will, on closing the contour, be dominated by the nearest singularities).

We turn now to the issue of imposing momentum cutoffs on the BFKL equation. The important point to note is that the effect of introducing an infra-red cutoff on the real graphs in eq.(2) is to remove the right-half plane poles of $\mathcal{F}(N, \omega)$ leaving only those in the left-half $\omega$-plane. To see this we use the following integral representation of the $\Theta$-function! [6]

$$
\Theta\left(k^{2}-Q_{0}^{2}\right)=\int_{c} \frac{d \mu}{2 \pi i} \frac{\left(k^{2} / Q_{0}^{2}\right)^{\mu}}{\mu} .
$$

Let us define some function, $f_{c}\left(x, k_{\perp}^{2}\right)$, which is non-zero only for $k_{\perp}^{2}>Q_{0}^{2}$, (hereafter subscript ' $c$ ' denotes a cutoff quantity) in terms of an unrestricted function, $g\left(x, k_{\perp}^{2}\right)$, by

$$
f_{c}\left(x, k_{\perp}^{2}\right) \equiv \Theta\left(k^{2}-Q_{0}^{2}\right) g\left(x, k_{\perp}^{2}\right) .
$$

By taking double Mellin transforms of both sides we obtain the equation

$$
\mathcal{F}_{c}(N, \omega)=\int_{c_{\nu}} \frac{d \nu}{2 \pi i} \frac{Q_{0}^{2(\nu-\omega)} \mathcal{G}(N, \nu)}{\omega-\nu}
$$

where we have used eq.(10) and changed variables from $\mu$ to $\nu=\omega-\mu$. For the cases of interest the asymptotic behaviour of $\mathcal{G}(N, \nu)$ is such that we can close

\footnotetext{
${ }^{1}$ The contour lies to the right of the pole at $\mu=0$ and is parallel to the imaginary axis. If $k^{2}>Q_{0}^{2}$, then the contour is closed to the left, we pick up the residue of the pole at $\mu=0$ and the result is 1 . If $k^{2}<Q_{0}^{2}$, then closure is to the right, where there are no poles, and the result is 0 .
} 
the contour via an infinite semicircle in either the left-half and/or the right-half $\nu$-plane with no contribution from the contour at infinity. If we take $\mathcal{G}(N, \nu)$ to have only simple poles in the $\nu$-plane then it follows that $\mathcal{F}_{c}(N, \omega)$ has poles only in the left-half plane (the right-half plane poles being projected out by the theta function which imposes the infra-red cutoff). Conversely for an ultraviolet cutoff only the right-half plane poles survive.

It will prove to be useful in solving the BFKL integral equation to find an integral representation for $\mathcal{F}_{c}(N, \omega)$. Since $f_{c}\left(x, k_{\perp}^{2}\right)=0$ for all $k^{2}<Q_{0}^{2}$, we may write

$$
f_{c}\left(x, k_{\perp}^{2}\right)=\Theta\left(k^{2}-Q_{0}^{2}\right) f_{c}\left(x, k_{\perp}^{2}\right)
$$

Thus we have

$$
\mathcal{F}_{c}(N, \omega)=\int_{c_{\nu}} \frac{d \nu}{2 \pi i} \frac{Q_{0}^{2(\nu-\omega)}\left(\mathcal{F}_{c}(N, \nu)-\mathcal{S}(N, \nu)\right)}{\omega-\nu}
$$

and we have made explicit the fact that we may add a function $\mathcal{S}(N, \nu)$ to the integrand of the right-hand side which has poles in the right-half $\nu$-plane (provided that $S(N, \nu)$ has the asymptotic behaviour which allows the contour to be closed in the left-half $\nu$-plane with no contribution from the semicircle at infinity).

In the presence of an infra-red cutoff on the real emission terms, the BFKL equation takes the form [6]:

$$
\begin{aligned}
\mathcal{F}_{c}(N, \omega) & =\mathcal{F}_{0, c}(N, \omega) \\
& +\int \frac{d \nu}{2 \pi i} \frac{Q_{0}^{2(\nu-\omega)}}{\omega-\nu}\left(\frac{-K(\nu)}{N}\right) \mathcal{F}_{c}(N, \nu)
\end{aligned}
$$

where $\mathcal{F}_{0, c}$ is the initial distribution with a cutoff. We can now solve this integral equation by subtracting it from eq.(14) to give

$$
\int \frac{d \nu}{2 \pi i} \frac{Q_{0}^{2(\nu-\omega)}}{\omega-\nu}\left(\mathcal{F}_{c}(N, \nu)-S(N, \nu)+\left(\frac{K(\nu)}{N}\right) \mathcal{F}_{c}(N, \nu)-\mathcal{F}_{0, c}(N, \nu)\right)=0
$$

Since we have taken care to add $\mathcal{S}$ to describe the ambiguities we may equate the integrand in the above equation to zero. Thus

$$
\left(1+N^{-1} K(\omega)\right) \mathcal{F}_{c}(N, \omega)=\mathcal{F}_{0, c}(N, \omega)+\mathcal{S}(N, \omega)
$$


i.e.

$$
\mathcal{F}_{c}(N, \omega)=\frac{1}{\left(1+N^{-1} K(\omega)\right)}\left(\mathcal{F}_{0, c}(N, \omega)+\mathcal{S}(N, \omega)\right) .
$$

Comparing to the non-cutoff solution eq.(7) we see that introducing the infra-red cutoff has two effects. Firstly, it converts the transform of the input solution to its cutoff equivalent (which induces a factor $Q_{0}^{-2 \omega}$ in $\mathcal{F}_{0, c}$ ), and secondly it introduces the function $\mathcal{S}$ whose right-half plane poles cancel those present in the original solution $\mathcal{F}$ leaving $\mathcal{F}_{c}(N, \omega)$ with only left-half plane poles (this happens for each term in the geometric series). Note that as $N^{-1} K(\omega)$ has only simple poles then $\mathcal{S}(N, \omega)$ will also have only simple poles, as required. Moreover the asymptotic behaviour of $\mathcal{S}(N, \omega)$ must include the factor $Q_{0}^{-2 \omega}$ to ensure the correct behaviour of the integrand at infinity.

The right-half plane poles of $\mathcal{S}$ must balance those of $N^{-1} K(\omega) \mathcal{F}_{c}$ on the lefthand side of eq.(17). If we keep $n$ right-half plane poles in eq.(9) then we will have $n$ conditions which will determine $\mathcal{S}$. Thus we may write

$$
\mathcal{S}(N, \omega)=\sum_{i}^{n} \frac{a_{i}^{\prime}}{\omega-\omega_{i}}
$$

where $\omega_{i}=1 / 2,3 / 2, \cdots,(2 n-1) / 2$ and the coefficients $a_{i}^{\prime}$ are determined by the cancellation of the right-half plane poles in the denominator of eq.(7). If $\left(1+N^{-1} K(\omega)\right)$ has zeros at $\omega=\omega_{s}$ then the $n$ conditions are

$$
\mathcal{F}_{0}\left(N, \omega_{s}\right)+\mathcal{S}\left(N, \omega_{s}\right)=0
$$

for the $n$ values of $s$. This procedure generates the solution to eq.(15).

Let us turn now to the case that there is both a lower and an upper cutoff on $k_{\perp}^{2 \prime}$. In this case the BFKL equation becomes

$$
\begin{aligned}
\mathcal{F}_{c}(N, \omega) & =\mathcal{F}_{0, c}(N, \omega) \\
& +\int \frac{d \nu}{2 \pi i} \frac{Q_{0}^{2(\nu-\omega)}-Q_{1}^{2(\nu-\omega)}}{\omega-\nu}\left(\frac{-K(\nu)}{N}\right) \mathcal{F}_{c}(N, \nu)
\end{aligned}
$$

where $Q_{1}^{2}$ is the upper cutoff. The solution to this equation proceeds in an analogous manner to that presented above. We introduce the integral representation

$$
\mathcal{F}_{c}(N, \omega)=\int_{c_{\nu}} \frac{d \nu}{2 \pi i} \frac{\left(Q_{0}^{2(\nu-\omega)}-Q_{1}^{2(\nu-\omega)}\right)\left(\mathcal{F}_{c}(N, \nu)-\mathcal{S}(N, \nu)\right)}{\omega-\nu} .
$$


As before, the function $S(N, \nu)$ can be any function that gives zero after the integration of eq.(22). Since the first term (in parentheses) of the integrand is zero at $\omega=\nu$ it follows that the function $\mathcal{S}(N, \nu)$ can have left-half (right-half) poles providing that the asymptotic behaviour of their associated functions allow the closure of the integration contour in the right-half (left-half) $\nu$-plane with zero contribution from the semicircle at infinity. In order to determine $\mathcal{S}(N, \nu)$ we recall that a function with a lower (upper) momentum cutoff has no poles in the right-half (left-half) $\nu$-plane. Consequently $\mathcal{F}_{c}$ with both lower and upper momentum cutoff must have no poles at all in the $\nu$-plane. Thus the function $\mathcal{S}(N, \nu)$ will be determined by the condition that it remove all the poles of $\mathcal{F}_{c}$. This procedure leads to the solution to eq.(21), again in the form of eq(18).

In general, if we keep $n$ right-half and $m$ left-half plane poles in $K(\omega)$ then we have $n+m$ conditions to determine the $n+m$ coefficients in $\mathcal{S}$. With

$$
\mathcal{S}(N, \omega)=\sum_{i}^{n} \frac{a_{i}^{\prime}}{\omega-\omega_{i}}+\sum_{j}^{m} \frac{b_{j}^{\prime}}{\omega+\omega_{j}}
$$

the conditions are the $n$ of eq.(20) together with $m$ new conditions given by

$$
\mathcal{F}_{0}\left(N,-\omega_{t}\right)+\mathcal{S}\left(N,-\omega_{t}\right)=0 .
$$

This completes the solution of the BFKL equation for the case of an upper and a lower momentum cutoff.

\section{$3 \quad$ Examples}

We now illustrate the method by considering two examples:

1. Infra-red cutoff including only the nearest poles in $K(\omega)$;

2. Infra-red and ultra-violet cutoffs with only the nearest poles kept in $K(\omega)$. We choose these two cases since they have been studied already by Collins \& Landshoff (see ref. [6]) and so we may compare the solutions derived by the two methods. Thus we take

$$
K(\omega)=\frac{3 \alpha_{s}}{\pi} \frac{4 \ln 2}{\left(1-4 \omega^{2}\right)}=\frac{K_{0}}{\left(1-4 \omega^{2}\right)} .
$$




\section{Example 1}

Introducing an infra-red cutoff on the BFKL equation removes the possibility of right-half plane poles in $\mathcal{F}_{c}$ (see eq.(17)). Since we know $\mathcal{F}_{c}$ and $\mathcal{F}_{0, c}$ have no poles in the right-half plane, the poles in $\mathcal{S}$ must cancel those present in the $N^{-1} K(\omega) \mathcal{F}_{c}$ term, i.e. $\mathcal{S}$ must have the same right-half plane pole as $K(\omega)$, so

$$
\mathcal{S}=\frac{a_{1}}{\omega-\frac{1}{2}}
$$

The only zero of $\left(1+N^{-1} K(\omega)\right)$ which lies in the right-half plane, for the choice eq. (25), is at $\omega=\omega_{N}$ where

$$
\omega_{N}=\frac{1}{2} \sqrt{1+\frac{K_{0}}{N}} .
$$

For $\mathcal{F}_{c}$ not to have right-half plane poles it follows that

$$
\mathcal{F}_{0, c}\left(N, \omega_{N}\right)+\mathcal{S}\left(N, \omega_{N}\right)=0
$$

The function $\mathcal{S}$ required for this cancellation is uniquely determined:

$$
\mathcal{S}(N, \omega)=\frac{\frac{1}{2}-\omega_{N}}{\omega-\frac{1}{2}} \mathcal{F}_{0, c}\left(N, \omega_{N}\right) Q_{0}^{-2\left(\omega-\omega_{N}\right)} .
$$

Note the inclusion of the factor $Q_{0}^{-2\left(\omega-\omega_{N}\right)}$ in $\mathcal{S}$ (which is unity when $\omega=\omega_{N}$ for consistency with eq.(26)). This gives $\mathcal{S}$ the correct asymptotic behaviour (i.e. giving zero contribution to the contour integral from the semicircle at infinity). Hence, we have for our cutoff solution

$$
\begin{aligned}
\mathcal{F}_{c}(N, \omega)= & \frac{1}{1+N^{-1} K(\omega)} \times \\
& \left(\mathcal{F}_{0, c}(N, \omega)+\frac{\mathcal{F}_{0, c}\left(N, \omega_{N}\right)\left(\frac{1}{2}-\omega_{N}\right) Q_{0}^{-2\left(\omega-\omega_{N}\right)}}{\omega-\frac{1}{2}}\right),
\end{aligned}
$$

which agrees with that derived in [6], i.e.

$$
\begin{aligned}
\mathcal{F}_{c}(N, \omega)= & \frac{1}{1+N^{-1} K\left(\omega_{0}\right)} \times \\
& \left(\mathcal{F}_{0, c}(N, \omega)+\frac{\mathcal{F}_{0, c}\left(N,-\frac{1}{2}\right)\left(\frac{1}{2}-\omega_{N}\right)}{\omega+\omega_{N}} Q_{0}^{-1-2 \omega}\right) .
\end{aligned}
$$




\section{Example 2}

In this example we introduce an ultra-violet, $Q_{1}^{2}$, and an infra-red cutoff, $Q_{0}^{2}$. Again, we keep only the nearest poles of the eigenvalues so that $K(\omega)$ takes the form of eq.(25). In this case, the ultra-violet cutoff projects out the remaining left-half plane poles of $\mathcal{F}_{c}$. We therefore insist that the function we add, $\mathcal{S}$, to the original solution cancels off poles in both the left-half and right-half of the $\omega$-plane. Since $\mathcal{S}$ must cancel the two poles of $K(\omega)$ it must be of the form

$$
\mathcal{S}(N, \omega)=\frac{a_{1}}{\omega-\frac{1}{2}}+\frac{b_{1}}{\omega+\frac{1}{2}} .
$$

The coefficients are determined by the fact that the poles in $\mathcal{F}_{c}$ associated with with the zeros of $\left(1+N^{-1} K(\omega)\right)$ are explicitly cancelled. We have two conditions

$$
\mathcal{F}_{0, c}\left(N, \pm \omega_{N}\right)+\mathcal{S}\left(N, \pm \omega_{N}\right)=0
$$

$\mathcal{S}(N, \omega)$ has terms with left-half (right-half) plane poles which have the appropriate asymptotic behaviour to ensure convergent closure of the contour in the right-half (left-half) $\nu$-plane. Thus, after including the appropriate factors $Q_{0}^{-2 \omega}$ or $Q_{1}^{-2 \omega}$, we arrive at the following form for the solution in the double-cutoff case

$$
\begin{aligned}
\mathcal{F}_{c}(N, \omega)= & \frac{1}{1+N^{-1} K(\omega)} \times \\
& {\left[\mathcal{F}_{0, c}(N, \omega)+\frac{1}{\Delta\left(\omega_{N}\right)}\left\{\Delta\left(\omega_{N}\right) \mathcal{S}(N, \omega)\right\}\right], }
\end{aligned}
$$

where

$$
\begin{aligned}
\Delta\left(\omega_{N}\right) \mathcal{S}(N, \omega) & =\frac{Q_{0}^{-2 \omega}}{\left(\omega-\frac{1}{2}\right)} \\
& \times\left[\left(\frac{1}{2}+\omega_{N}\right) Q_{1}^{2 \omega_{N}} \mathcal{F}_{0, c}\left(N, \omega_{N}\right)-\left(\frac{1}{2}-\omega_{N}\right) Q_{1}^{-2 \omega_{N}} \mathcal{F}_{0, c}\left(N,-\omega_{N}\right)\right] \\
& +\frac{Q_{1}^{-2 \omega}}{\left(\omega+\frac{1}{2}\right)} \\
& \times\left[\left(\frac{1}{2}-\omega_{N}\right) Q_{0}^{2 \omega_{N}} \mathcal{F}_{0, c}\left(N, \omega_{N}\right)-\left(\frac{1}{2}+\omega_{N}\right) Q_{0}^{-2 \omega_{N}} \mathcal{F}_{0, c}\left(N,-\omega_{N}\right)\right] .
\end{aligned}
$$

This may be rewritten

$$
\begin{aligned}
\mathcal{F}_{c}(N, \omega)= & \frac{1}{1+N^{-1} K\left(\omega_{0}\right)} \times \\
& \left(\mathcal{F}_{0, c}(N, \omega)-G\left(\omega, \omega_{N}\right)-G\left(\omega,-\omega_{N}\right)\right),
\end{aligned}
$$


with

$$
\begin{aligned}
& G\left(\omega, \omega_{N}\right)=\frac{\left(Q_{0}^{2\left(\omega_{N}-\omega\right)}-Q_{1}^{2\left(\omega_{N}-\omega\right)}\right) C\left(\omega_{N}\right)}{\omega-\omega_{N}} \\
& \Delta\left(\omega_{N}\right) C\left(\omega_{N}\right)=\frac{\left(\frac{1}{2}+\omega_{N}\right) Q_{0}^{1-2 \omega_{N}} \mathcal{F}_{0, c}\left(N, \frac{1}{2}\right)}{R^{-2 \omega_{0}}-R^{-1}} \\
& -\frac{\left(\frac{1}{2}-\omega_{N}\right) Q_{1}^{-1-2 \omega_{N}} \mathcal{F}_{0, c}\left(N,-\frac{1}{2}\right)}{R^{2 \omega_{0}}-R^{-1}}, \\
& \Delta\left(\omega_{N}\right)=\frac{\frac{1}{2}+\omega_{N}}{\frac{1}{2}-\omega_{N}} R^{2 \omega_{N}}-\frac{\frac{1}{2}-\omega_{N}}{\frac{1}{2}+\omega_{N}} R^{-2 \omega_{N}}
\end{aligned}
$$

where $R=\frac{Q_{1}}{Q_{0}}$. In contrast, Collins and Landshoff found

$$
\begin{aligned}
\mathcal{F}_{c}(N, \omega)= & \frac{1}{1+N^{-1} K\left(\omega_{0}\right)} \times \\
& \left(\mathcal{F}_{0, c}(N, \omega)+G\left(\omega, \omega_{N}\right)+G\left(\omega,-\omega_{N}\right)\right)
\end{aligned}
$$

The two solutions (eq.(32) and eq.(33)) differ only in a relative minus sign and we have checked explicitly (by direct substitution into the integral equation) that eq.(32) is the correct solution. Furthermore, by taking the limit $Q_{1} \rightarrow \infty$ in eq.(32) we arrive at eq.(28) (this limit is not correctly reproduced from eq.(33)).

\section{The solution in $\left(x, k_{\perp}^{2}\right)$-space}

Having produced a double cutoff solution $\mathcal{F}_{c}(N, \omega)$ that is free from poles one may inquire how it is that a non-zero gluon distribution arises upon inverting back to $\left(x, k_{\perp}^{2}\right)$-space. The key to understanding this lies in the inclusion of the $Q_{0}^{-2 \omega}$ and $Q_{1}^{-2 \omega}$ factors in $\mathcal{S}$. Consider the two terms in eq. 31) separately. Upon substituting $\mathcal{F}_{c}(N, \omega)$ in the inverse transform of eq.(4) the first term forces the contour, $c_{\omega}$, to be closed to the left (since $k_{\perp}^{2} / Q_{0}^{2}>1$ ). On the other hand, the second term must be closed to the right (since $k_{\perp}^{2} / Q_{1}^{2}<1$ ). In this way a nonzero function is formed. It is easier in this case, however, to invert eq.(32) and arrive at

$$
\begin{aligned}
\mathcal{F}_{c}\left(N, k_{\perp}^{2}\right)= & \frac{-1}{1+N^{-1} K\left(\omega_{0}\right)} \times \\
& \left(\frac{A}{N+\epsilon}\left(k_{\perp}^{2}\right)^{\frac{1}{2}+\omega_{0}}+C\left(\omega_{N}\right)\left(k_{\perp}^{2}\right)^{\frac{1}{2}+\omega_{N}}+C\left(-\omega_{N}\right)\left(k_{\perp}^{2}\right)^{\frac{1}{2}-\omega_{N}}\right),
\end{aligned}
$$


for $Q_{0}<k_{\perp}<Q_{1}$ and 0 otherwise. Note this has an overall minus sign when compared to eq. (22) of [6].

We now discuss the structure of the solution in the N-plane and the corresponding small- $x$ behaviour of the gluon density, for the various cases.

In the non-cutoff case we have two poles at $\omega= \pm \omega_{N}$. The contour, $c_{\omega}$, is pinched as these two poles move together (corresponding to locating the nearest singularity in the $N$-plane) and coincide at $\omega=0$. The $\omega$-plane contour in eq.(3) is closed and this removes one of these poles to leave a $1 / \sqrt{N+K_{0}}$ singularity in the $N$-plane. The $N$-plane contour is then closed and the discontinuity along this cut is taken. This leads to the $x^{-K_{0}} \ln ^{-1 / 2}(1 / x)$ behaviour (where $K_{0} \simeq 1 / 2$ for $\alpha_{s} \simeq 0.2$ (see eq.(25)).

In the case of an infra-red cutoff we close the contour, $c_{\omega}$, to the left and, picking up the residue of the pole at $\omega=-\omega_{N}$, we are left with the $(1 / 2-$ $\left.\omega_{N}\right)$ terms explicit in eqs.(27, 28) which then lead to a $\sqrt{N+K_{0}}$ cut in the $N$ plane. The discontinuity across this cut leads to a $x^{-K_{0}} \ln ^{-3 / 2}(1 / x)$ dependence. So introducing an infra-red cutoff has made only a small difference to the $x$ dependence of the solution.

In the double cutoff case things are very different. The singularities in the $N$-plane are at the zeros of $\Delta\left(\omega_{N}\right)$ see eqs. 30, 32) (with the exception of $\omega_{N}=0$ which cancels). This leads to a series of poles in the $N$-plane; the locations of which are determined by solving the equation $\Delta\left(\omega_{N}\right)=0$ and depend strongly upon the value of $R$. The roots of this equation correspond to pure imaginary values of $\omega_{N}$. With $\bar{\omega}_{N}=\Im m\left(\omega_{N}\right)$ the roots then satisfy the following equations:

$$
\begin{aligned}
\bar{\omega}_{N} & =\frac{1}{2} \cot \left(\bar{\omega}_{N} \ln R\right), \text { and }, \\
\bar{\omega}_{N} & =-\frac{1}{2} \tan \left(\bar{\omega}_{N} \ln R\right) .
\end{aligned}
$$

For large $R$, the solutions to these transcendental equations occur near the zeros of the periodic functions and so we obtain approximate solutions by assuming a linear approximation to these functions about their zeroes. This leads to the solutions

$$
\bar{\omega}_{N} \approx \frac{n \pi / 2}{2+\ln R}
$$


where $n$ is a positive integer. The corresponding positions of the poles in the $N$-plane are given by

$$
N=-K_{0}\left(\frac{1}{1+4 \bar{\omega}_{N}^{2}}\right) \approx \frac{-K_{0}}{\left(1+\frac{n^{2} \pi^{2}}{(2+\ln R)^{2}}\right)}
$$

The leading pole occurs for $n=1$ in eq.(38) and for $R=100$ is $82 \%$ of the asymptotic value (indeed it only falls to $65 \%$ for $R$ as small as 10). We therefore disagree with the conclusion of ref. [6] where the claim is that only for $R$ as big as $10^{4}$ does the position of the leading singularity lie within $10 \%$ of $-K_{0}$ and therefore that the necessary cutoffs on the transverse momentum integrals have a 'very significant moderating effect' on the small- $x$ behaviour. The origin of the discrepancy is clear and is merely a result of the approximations used in ref. [6] when extracting the solutions to eq.(35).

The next-to-leading singularity $(n=2)$ is determined by the solution to eq.(36) and can lie close to the leading singularity for moderate values of $R$, e.g. for $R=100$ it is $52 \%$ of $-K_{0}$. Of course as $R \rightarrow \infty$ all the poles merge together and form the original square root branch cut. We note that Collins \& Landshoff did not consider the solutions to eq.(36) and consequently missed the location of this pole.

\section{Summary}

We have presented an analytic solution to the BFKL equation with infra-red and/or ultra-violet cutoffs on the transverse momentum for real gluon emission. Our method is applicable to the general case where an arbitrary number of poles in the kernel, $K(\omega)$, are included and applies for any functional form for the input distribution. Since our method leads to an expression for the solution in terms of a geometric series (eqs.(7,,18)) it may be modified to allow for a summation over the emission of a finite number of gluons [9]. This should allow a more realistic implementation of energy-momentum conservation. \& We have verified

\footnotetext{
${ }^{2}$ We thank Peter Landshoff for pointing out the interest in such a restricted sum.
} 
the result of Collins \& Landshoff in the case of an infra-red cutoff and correct their result in the case of both infra-red and ultra-violet cutoffs. We find that the imposition of both cutoffs reduces the small $x$ rise of the gluon density but not by the amount claimed in ref. [6]. In particular, for an infra-red cutoff of $1 \mathrm{GeV}$ and an ultra-violet cutoff equal to the typical HERA $\gamma p$ centre-of-mass energy, i.e. $\sim 100 \mathrm{GeV}$, we expect a leading power behaviour like $\sim x^{-0.4}\left(\right.$ for $-K_{0}=0.5$ ). This behaviour is consistent with current extractions of the gluon density from HERA [2, 10].

\section{References}

[1] ZEUS Collaboration, Phys. Lett. B316 (1993) 412; DESY 94-143; H1 collaboration, Nucl.Phys. B407 (1993) 515.

[2] G. Wolf, HERA Physics, DESY 94-022 (1994).

[3] R.D. Ball, S. Forte, Phys. Lett. B335 (1994) 77; Phys. Lett. B336 (1994) 77; M. Gluck, E.Reya and A.Vogt, Phys. Lett. B306 (1993) 391; R.K.Ellis, Z.Kunszt and E.M.Levin, Nucl.Phys. B420 (1994) 517.

[4] E.A. Kuraev, L.N. Lipatov and V.Fadin, Zh. Eksp. Teor. Fiz 72 (1977) 373; Sov. Phys. JETP 45 (1977) 199; Ya.Ya. Balitskij and L.N.Lipatov, Yad.Fiz. 28 (1978) 1597; Sov. J. Nucl. Phys. 28 (1979) 822; L.N.Lipatov, in "Perturbative QCD”, ed. A.H. Mueller (World Scientific, Singapore, 1989) 411.

[5] A.J. Askew, J. Kwiecinski, A.D. Martin and P.J. Sutton, Phys. Rev. D47 (1993) 3775; Phys. Rev. D49 (1994) 4402.

[6] J.C. Collins and P.V.Landshoff, Phys. Lett. B276 (1992) 196.

[7] J.R. Forshaw, P.N. Harriman and P.J. Sutton, J.Phys G19 (1993) 1616; Nucl. Phys. B416 (1994) 739.

[8] R.E. Hancock and D.A. Ross, Nucl. Phys. B383 (1992) 575.

[9] J.R.Forshaw, M.F.McDermott and G.G.Ross, in preparation. 
[10] H1 Collaboration, Phys. Lett. B321 (1994) 161; ZEUS Collaboration, DESY 94-192 (1994). 